\title{
¿Evaluamos la calidad de la evidencia antes de incluir una práctica médica?
}

Do we assess the quality of evidence before including a medical practice?

Objetivo

Identificar prácticas médicas que no ofrecen beneficios.

\section{Fuentes de datos}

Revisión de todos los artículos clasificados como "artículos originales", publicados en New England Journal of Medicine (NEJM) entre los años 2001 y 2010.

\section{Selección de estudios}

Artículos originales publicados en NEJM referidos a prácticas médicas.

\section{Extracción de datos}

Los datos fueron extraídos por dos revisores independientes. Detectaron los artículos originales que se referían a prácticas médicas. Luego los clasificaron en cuatro categorías: reemplazo, de vuelta a la base, inversión y reafirmación. Ver tabla 1.

Tabla 1. Clasificación de los artículos según los resultados y la antigüedad de la práctica

\begin{tabular}{l|c|c}
\multicolumn{1}{c|}{ CLASIFICACIÓN } & Conclusión negativa & Conclusión positiva \\
\hline Práctica nueva & De vuelta a la base & Reemplazo \\
\hline Práctica establecida & Inversión & Reafirmación \\
\hline
\end{tabular}

\section{Resultados Principales}

Se revisaron 2.044 artículos originales, de los cuales 1.344 correspondían a prácticas médicas. De las mismas 981 (73\%) se referían a prácticas nuevas y $363(27 \%)$ a prácticas establecidas. Por otro lado 947 estudios $(70,5 \%)$ presentaron resultados positivos, mientras $397(29,5 \%)$ alcanzaron resultados nega-
Waters D y col. J. Am. Coll. Cardiol. 2013; 61:148-52.

tivos. De los 363 estudios sobre prácticas establecidas, 146 $(40,2 \%)$ indicaban inversión de la práctica y 138 (38\%) la reafirmaban. Dentro de estas se incluyeron medicaciones, procedimientos, estudios diagnósticos, test de rastreo y monitoreo y dispositivos de tratamiento. Los resultados principales se detallan en la tabla 2.

Tabla 2. Número (porcentaje) de artículos por año con resultados de inversión, reafirmación o inconclusos.

\begin{tabular}{c|l|c|c} 
Año & Inversión & Reafirmación & Inconclusos \\
$\mathbf{2 0 0 1}(\mathbf{n}=\mathbf{4 8})$ & $14(29,2)$ & 20 & 14 \\
\hline $\mathbf{2 0 0 2}(\mathbf{n}=\mathbf{2 6})$ & $12(46,2)$ & 9 & 5 \\
\hline $\mathbf{2 0 0 3}(\mathbf{n}=\mathbf{3 1})$ & $12(38,79)$ & 12 & 7 \\
\hline $\mathbf{2 0 0 4}(\mathbf{n}=\mathbf{3 3})$ & $12(36,4)$ & 15 & 6 \\
\hline $\mathbf{2 0 0 5}(\mathbf{n}=\mathbf{4 1})$ & $19(46,3)$ & 14 & 8 \\
\hline $\mathbf{2 0 0 6}(\mathbf{n}=\mathbf{2 0})$ & $12(60,0)$ & 5 & 3 \\
\hline $\mathbf{2 0 0 7}(\mathbf{n}=\mathbf{5 4})$ & $18(33,3)$ & 17 & 19 \\
\hline $\mathbf{2 0 0 8}(\mathbf{n}=\mathbf{3 2})$ & $15(46,9)$ & 13 & 4 \\
\hline $\mathbf{2 0 0 9}(\mathbf{n}=\mathbf{3 5})$ & $16(45,7)$ & 16 & 3 \\
\hline $\mathbf{2 0 1 0}(\mathbf{n}=\mathbf{4 2})$ & $16(37,2)$ & 17 & 10 \\
\hline Total (N=363) & $146(40,2)$ & $138(38,0)$ & $79(21,7)$ \\
\hline
\end{tabular}

\section{Conclusiones}

La presencia de prácticas médicas establecidas cuya revisión muestra resultados negativos (inversión) es común y ocurre en toda clase de áreas y prácticas médicas.

Fuente de financiamiento/Conflicto de interés de los autores: no referidos.

\section{Comentario}

El presente artículo realizó una revisión de publicaciones presentadas en una revista de alto impacto durante 10 años. Se examinaron 2.044 artículos, identificándose 146 artículos sobre prácticas médicas establecidas con resultados negativos (inversión de la práctica médica); mostrando que un alto porcentaje de todas las prácticas podrían no ser efectivas. El objetivo no fue desaconsejarlas, sino ponerlas en duda.

La pregunta que se plantea entonces, es como llegan las mismas a establecerse. Para dicha hipótesis se plantea la opción de que muchas de ellas se basan en evidencia prematura, inadecuada y sesgada. Esto conlleva un alto riesgo de resultados erróneos y un gran costo para la sociedad y el sistema médico. Este estudio avala la necesidad de aumentar el nivel requerido para la aprobación de prácticas médicas y la impor- tancia de contar con evidencia de alta calidad previa a la divulgación de las mismas.

Los resultados de este estudio concuerdan con una revisión que demuestra que el $15 \%$ de las prácticas son dañinas, raramente beneficiosas y que el $50 \%$ tienen una efectividad desconocida'.

Dentro de las limitaciones del estudio es importante destacar la elección de la revista, el posible sesgo de publicación, favoreciendo la publicación de ciertos estudios, principalmente aquellos que contradicen prácticas. Por otro lado, no se realizó una búsqueda independiente para constatar si las prácticas actuales estaban realmente en uso y las prácticas nuevas no, corriendo el riesgo de errores en inclusión y exclusión.

María Teste [ Servicio de Medicina Familiar y Comunitaria del Hospital Italiano de Buenos Aires. maria.teste@ hospitalitaliano.org.ar ]

Teste M. ¿Evaluamos la calidad de la evidencia antes de incluir una práctica médica? Evid Act Pract Ambul. Ene-Mar 2014;17(1):18. Comentado de: Prasad V, Vandross A, Toomey C, y col. A decade of reversal: An analysis of 146 contradicted medical practices. Mayo Clinic Proceedings 2013;88(8):790-798. PMID: 23871230.

Referencia

1.What conclusions has Clinical Evidence drawn about what works, what doesn't based on randomised controlled trial evidence?

http://clinicalevidence.bmj.com/x/set/static/cms/efficacycategorisations.html. Accessed June 30, 2011. 